\title{
A social movement to reduce caries prevalence in the world
}

\section{Marcelo Bönecker(a) Gilberto Alfredo Pucca Junior ${ }^{(b)}$ Patricia Bella Costa(c) Nigel Pitts ${ }^{(\mathrm{d})}$}

\footnotetext{
(a) Department of Pediatric Dentistry, School of Dentristry, Univ de São Paulo, São Paulo, SP, Brazil.

(b) Ministério da Saúde, Brasília, DF, Brazil.

(c) Colgate-Palmolive, Brazil.

(d) Dental Health Services Research Unit - Univ of Dundee, Scotland.
}

D espite the fact that it is preventable, dental caries remains a global public health problem-even with fluoride intervention-and it is the most common chronic disease on the planet. Moreover, researchers have already shown for some years that caries is a disease continuum that at the early stages is reversible, yet many Ministries of Health and dental professionals misunderstand this.

We believe it's important to address these issues and to drive broader understanding and action. As such, a public health program, the Alliance for a Cavity-Free Future (http://www.allianceforacavityfreefuture.org/en/us/aboutus), was developed. In the history of dentistry we have never yet had effective engagement between healthcare professionals, the government and other stakeholders to contain this widespread problem of dental caries in the world.

The Alliance is a social movement, which convenes a worldwide group of experts who have joined together to work towards the goal of a Cavity-Free Future for all age groups. It acknowledges the global problem of caries, promotes an understanding of caries as a disease continuum, which is reversible, and will drive action to move communities and individuals to make the changes necessary to achieve the goal of a Cavity-Free Future.

Overall, the group believes that global collaborative action is needed to challenge global leaders and other regional and local stakeholders to learn the importance of caries as a disease continuum and to participate in action toward the delivery of comprehensive caries prevention and management that can positively influence the continuing problem of caries.

At the global level, the Alliance was launched on September 3, 2011 at the FDI World Dental Federation Congress in Salvador, Brazil. Since then the Expert Panel and Co-directors have been working hard to develop this initiative and have already received a tremendous amount of positive feedback from the global dental and public health community.

The Alliance has identified the following long-term goals:

- By 2015, ninety percent of dental schools and dental associations should have embraced and promoted the "new" approach of "caries as a continuum" to improve dental caries prevention and management.

- By 2020, regional members (Chapters) of the Alliance for a Cavity-Free Future should have integrated, locally appropriate, comprehensive caries prevention and management systems and monitoring developed and in place.

- Every child born in 2026 should stay cavity free during their life time.

In Brazil, the local Alliance Chapter was launched on January 29, 2012 during the Sao Paulo International Dental Congress (CIOSP). There are 6 principles for the Alliance Chapter that were developed by a group of Brazilian experts supported by the Ministry of Health.

The project to tackle dental caries in Brazil, in spite of showing a clear success of the public policies based on the use of water containing fluoride, an increase in access to fluoride toothpaste and preventive programs based on using fluorides directed to school children, presents unequal access to those policies in the national geographic scenario. That inequality, associated to some precarious socioeconomic situations, is demonstrated in the epidemiology of sev- 
eral diseases, the caries disease among them. Therefore, based on the best scientific evidence currently available for the control of this disease, the Alliance for a Cavity Free Future (ACFF) states as follows:

1. Dental caries is a chronic disease resulting from an association between a necessary factor, accumulation of dental biofilm (plaque), and exposure to sugars (negative determining factor). Fluoride has an important role in its control by acting as a positive determining factor to reduce mineral loss when available in the oral cavity. Considering the modern societies' diets, caries is not eradicable, but it can be maintained under control without the appearance of advanced lesions during an individual's life, and the strategies currently available for that purpose involve controlling the necessary and determining causal factors (dental biofilm and diet) in addition to the use of fluorides.

2. The epidemiological scenario in Brazil demonstrates different caries prevalences both at the macro-regional level (the northern, northeastern and centerwestern regions have higher prevalence than the southern and southeastern regions) and at the local level (low-income regions inside the same city have higher prevalence of caries), showing an important social factor involved in the disease related to access to education and control strategies, thus characterizing caries as a bio-social disease.

3. The addition of fluoride to the public water supply provided in the Brazilian law is an important public health strategy to control caries, but there are differences in the caries prevalence in regions with or without access to water containing fluoride. So, the inequality in the access to water containing fluoride, in addition to its being one of the consequences of socioeconomic differences among the Brazilian regions, becomes an important factor in the control of the disease. The Alliance for a Cavity Free Future strongly recommends expanding the addition of fluoride in the water for public supply to cities which still do not have that benefit and stresses the need of heterocontrol of fluoride levels and of monitoring fluoride residual contents (in natura) in the water in order to guarantee maximum benefit with minimum risk of dental fluorosis.

4. The use of fluoride-containing dentifrices to control caries is based on scientific evidence and is recommended all over the world to individuals of all ages. Due to concerns about dental fluorosis resulting from the disseminated use of fluorides, it is recommended that a small quantity of fluoride-containing dentifrice be used daily by young children, thus guaranteeing its anti-caries benefit and minimum risk of having them develop dental fluorosis; to this end, providing more detailed guidance by members of the Oral-Health Professional Team is advisable.

5. Preventive programs based on the use of fluoride should be expanded to regions/communities at risk, which lack access to those strategies, maximizing cost efficiency.

6. The World Health Organization advises, as a global policy to improve oral health, that an action plan to promote health should be set as part of the integrated prevention against diseases. Converging towards the benefits from reaching that goal, the Alliance for a Cavity Free Future states that it is advisable that educational programs including the promotion of oral health as part of general health be continuously expanded by prioritizing groups at social risk of developing diseases, targeting on improving oral and general health. Those programs should be integrated to already existing strategies, such as the Family-Health Program, or regional educational programs for oralhealth education, thus enabling an expansion in the knowledge of dental caries prevention in geometric scale where dentists, oral-health technicians, community health agents, teachers and school children could act as multiplying agents in order to reach the Alliance for a Cavity Free Future's macro-objective.

Since the Alliance Chapter in Brazil was launched, 23\% of Dental Schools and Dental Associations were reached and are now promoting ACFF, 42 Bright Smile Centers were implemented in Dental Schools, 4 Symposia on Cariology were held in National Congresses and an Alliance website in Portuguese has been developed.

The next steps the Brazilian Chapter will achieve are to implement training on Oral Health for Community Agents from the Ministry of Health, contact food industries and others to join the Alliance and involve healthrelated professional associations.

In Latin America, the Alliance was also launched in Venezuela and Colombia. There are different ways that the Alliance Chapters have been built in different regions of the continent; however, the interaction between these 3 countries is allowing them to learn from each other through sharing their experiences. We hope that more countries will join this social movement in order to reduce the caries prevalence we have in our continent. 